\title{
Spintronic properties of carbon-based one-dimensional molecular structures
}

\author{
E. Durgun, ${ }^{1}$ R. T. Senger, ${ }^{1}$ H. Sevinçli, ${ }^{1}$ H. Mehrez, ${ }^{2}$ and S. Ciraci ${ }^{1}, *$ \\ ${ }^{1}$ Department of Physics, Bilkent University, 06800 Ankara, Turkey \\ ${ }^{2}$ Center for NanoTechnology and NASA Advanced Supercomputing Division, NASA Ames Research Center, Mail Stop 229-1, \\ Moffett Field, California 94035-1000, USA
}

(Received 19 July 2006; revised manuscript received 30 September 2006; published 8 December 2006)

\begin{abstract}
In this paper we present an extensive study of the electronic, magnetic, and transport properties of finite and infinite periodic atomic chains composed of carbon atoms and $3 d$ transition metal (TM) atoms using firstprinciples methods. Finite-size, linear molecules made of carbon atomic chains caped with TM atoms, i.e., TM- $\mathrm{C}_{n}$-TM structures are stable and exhibit interesting magnetoresistive properties. The indirect exchange interaction of the two TM atoms through a spacer of $n$ carbon atoms determines the type of the magnetic ground state of these structures. The $n$-dependent ( $n=1$ to 7$)$ variations of the ground state between ferromagnetic and antiferromagnetic spin configurations exhibit several distinct forms, including regular alternations for $\mathrm{Ti}, \mathrm{V}, \mathrm{Mn}, \mathrm{Cr}, \mathrm{Fe}$, and $\mathrm{Co}$, and irregular forms for $\mathrm{Sc}$ and $\mathrm{Ni}$ cases. We present a simple analytical model that can successfully simulate these variations, and the induced magnetic moments on the carbon atoms. Depending on the relative strengths of the carbon $s, p$ and TM $d$ orbital spin-dependent coupling and on the on-site energies of the TM atoms there induces long-range spin polarizations on the carbon atoms which mediate the exchange interaction. While periodically repeated $\mathrm{TM}-\mathrm{C}_{n}$ atomic chains exhibit half-metallic properties with perfect spin polarization at the Fermi level, finite but asymmetric chains comprising single, double, and triple TM atoms display interesting spin-dependent features. These properties may be altered when these structures are coupled to electrodes. However, when connected to appropriate electrodes the $\mathrm{TM}-\mathrm{C}_{n}-\mathrm{TM}$ atomic chains act as molecular spin valves in their ferromagnetic states due to the large ratios of the conductance values for each spin type.
\end{abstract}

DOI: 10.1103/PhysRevB.74.235413

PACS number(s): 73.63.Nm, 73.22.-f, 75.75.+a

\section{INTRODUCTION}

Utilizing the spin degree of freedom of electrons in the solid-state electronics has led to the emergence of a rapidly developing field of spintronics. ${ }^{1-3}$ Creation of nonequilibrium spin populations and spin-polarized currents are essential for spintronic device applications. Important electronic applications based on magnetoresistive effects in twodimensional (2D) heterostructures are already realized. ${ }^{4-6}$ Typical devices, such as magnetic read heads in computer hard drives, and nonvolatile magnetic random access memories are made of magnetic multilayers, where the relative alignment of the layer magnetizations causes large variations in the resistance of the structure. The effect is known as giant magnetoresistance (GMR), and was discovered in $\mathrm{Fe} / \mathrm{Cr}$ multilayers. ${ }^{7}$ In such magnetic superlattice structures, the magnetization of the layers are coupled to each other by an indirect exchange interaction mediated by the electrons of the spacer layer. ${ }^{8,9}$ The interlayer exchange coupling and the magnetoresistance are found to be oscillating as a function of the spacer thickness, and the interaction amplitude asymptotically decays proportional to the inverse square of the spacer thickness. ${ }^{10-12}$

There are continuing efforts in improving issues in materials fabrication and device design of layered magnetic structures. With the advent of nanotechnology fabrication of quantum structures with dimensions of the order of molecular and atomic sizes became accessible, and analogous magnetoresistive properties are studied in 1D geometry. In carbon nanotubes, for instance, the indirect exchange coupling between magnetic impurities are quite long ranged, ${ }^{13,14} \mathrm{a}$ property that can be exploited in future spintronic devices. Fundamental spin-dependent electron transport properties have been demonstrated in the context of molecular spintronics ${ }^{15-20}$ which is a promising field of research in basic science and potential applications. Even the ultimately thin wires made of single atomic chains are produced under experimental conditions and are actively studied. These nanowire systems include atomic chains of both metal and transition metal elements such as $\mathrm{Al}, \mathrm{Au}, \mathrm{Cr}, \mathrm{Fe}$, etc., as well as $\mathrm{C}$ and $\mathrm{Si}$ atomic chains which also exhibit metallic properties. ${ }^{21-31}$ Much recently, finite or periodic forms of transition metal (TM) monatomic chains have been subject of various theoretical studies. The atomic structure, and magnetic and transport properties of these chains have been investigated. ${ }^{32-35}$

In this context, first-principles studies of elemental or compound atomic chain structures that can produce spin polarization effects are important. Even the extreme case of complete spin polarization in the absence of magnetic field can be achieved in a special class of materials, the so-called half-metallic ferromagnets. ${ }^{36,37}$ Zinc-blende (ZB) half-metals with high magnetic moment $\mu$ and high Curie temperature $T_{c}>400 \mathrm{~K}$ (such as CrAs and $\mathrm{CrSb}$ in $\mathrm{ZB}$ structure) have been grown in thin-film forms. ${ }^{38}$ Density functional calculations show that CrAs-MnAs superlattices have half-metallic property with $100 \%$ spin-polarized electrons around the Fermi level. ${ }^{39}$ Half-metallic properties have been also predicted in simple 1D atomic chains composed of carbon and TM compounds. ${ }^{40}$ Carbon chains in this respect are promising, since carbon has a strong tendency to form linear atomic chains, whereas other elements tend to make zigzag chains and they are more vulnerable to clustering. ${ }^{30}$ In any real 
device the system length is certainly finite, so that the considerations regarding the properties of finite carbontransition-metal compound atomic chains, such as whether spin-polarized currents can be achieved in these structures, and the effects of metallic electrodes on their transport properties are interesting points to address.

In this paper we study the spin-polarized structural, magnetic, and electronic transport properties of carbon atomic chains caped with $3 d$ TM atoms. The linear structure of these finite atomic chains are stable even at elevated temperatures. The magnetic ground state of the TM- $\mathrm{C}_{n}-\mathrm{TM}$ molecular chains are either ferromagnetic (FM) or antiferromagnetic (AF) depending on the relative alignment of the local magnetic moments on the TM atoms. Whether the FM or AF alignment leads to a lower energy depends on the indirect exchange interaction of the TM atoms mediated by the carbon atomic chain. The ground state configuration and the total magnetic moment of the structures are determined by the number of carbon atoms in the spacer. The sizedependent variations of the physical properties of such systems exhibit several distinct forms, including regular alternations for $\mathrm{Ti}, \mathrm{V}, \mathrm{Mn}, \mathrm{Cr}, \mathrm{Fe}$, and $\mathrm{Co}$, and some irregular forms for $\mathrm{Sc}$ and $\mathrm{Ni}$ cases. In order to understand the underlying mechanism of such diverse variations we present a simple Hückel-type tight-binding model. We also investigate the transport properties of these structures. The conductance of TM- $C_{n}$-TM molecules when connected to metallic electrodes show a strong spin-valve effect.

\section{METHOD OF CALCULATIONS}

We have performed first-principles plane-wave calculations ${ }^{41,42}$ within density functional theory (DFT) ${ }^{43}$ using ultrasoft pseudopotentials. ${ }^{44}$ The exchange correlation potential has been approximated by spin polarized generalized gradient approximation (GGA) ${ }^{45}$ using PW91 functional. Other exchange-correlation potentials have been tested for comparison. All structures have been treated by supercell geometries using the periodic boundary conditions. To prevent interactions between adjacent structures a large spacing $(\sim 10 \AA)$ has been taken in all directions. In the selfconsistent potential and total energy calculations Brillouin zone (BZ) has been sampled by considering $\Gamma$ point only, since we are dealing with finite molecular systems. A planewave basis set with kinetic energy cutoff $\hbar^{2}|\mathbf{k}+\mathbf{G}|^{2} / 2 m$ $=350 \mathrm{eV}$ has been used. All atomic positions are optimized by using the conjugate gradient method where total energy and atomic forces are minimized. The convergence of calculations are achieved when the difference of the total energies of two consecutive steps is less than $10^{-5} \mathrm{eV}$ and the force on each atom is reduced below $0.05 \mathrm{eV} / \AA$. Magnetic ground state is identified by calculating the energy difference $\Delta E_{T}=E^{s u}-E_{T}^{s r}$ [the difference between spin-unpolarized (su) and spin-relaxed (sr) total energies]. Chains with $\Delta E_{T}>0$ and $\mu>0$ have ferromagnetic ground state. For antiferromagnetic states, $\Delta E_{T}>0, \mu=0$, but the sum of the absolute value of spin states, $\Sigma_{i}\left[\left|S_{i} \uparrow\right|+\left|S_{i} \downarrow\right|\right]>0$.

The stability of optimized structures at $T=0 \mathrm{~K}$ is tested first by applying deformations by displacing the atoms in random directions from their equilibrium positions and then by reoptimizing the deformed structures. Strongly deformed structures returning to their initial optimized configuration are taken stable. Furthermore, we carried out calculations on the vibrational modes of the structures to search for probable instabilities. In addition, we performed $a b$ initio molecular dynamics calculations at high temperatures using Langevin thermostat. ${ }^{46}$

It should be noted that for comparison purposes the calculations have been repeated by using methods which utilize local basis sets, such as SIESTA ${ }^{47}$ and GAUSSIAN03. ${ }^{48}$ In the GAUSSIAN03 calculations the UBPW91 and UB3LYP functionals with $6-31 \mathrm{G}^{* *}$ basis set have been employed and results have been subjected to wave function stability analysis. All these calculations have led to consistent results.

The equilibrium conductance calculations of the structures when connected to metallic electrodes have been done using Landauer formalism. ${ }^{49}$ The required Hamiltonian matrices of the device and electrode regions are obtained in a DZP basis (double-zeta basis plus polarization orbitals) using SIESTA, ${ }^{47}$ and the surface Green function of the electrodes is calculated recursively. ${ }^{50}$

\section{ATOMIC STRUCTURE}

\section{A. Binding energy and stability}

Carbon atom strings or chains, $\mathrm{C}_{n}$, which are the precursor to $\mathrm{TM}-\mathrm{C}_{n}-\mathrm{TM}$ molecular structures, have been investigated for decades ${ }^{51}$ Finite segments of $\mathrm{C}_{n}$ have already been synthesized ${ }^{52,53}$ As an ultimate 1D structure having only one atom in the cross section, carbon strings can form only linear atomic chains and are stabilized by double bonds, which consist of a $\sigma$ bond of the carbon $2 s+2 p_{z}$ atomic orbitals along the chain axis, and $\pi$ bonds of $2 p_{x}$ and $2 p_{y}$ orbitals. Because of the cylindrical symmetry of the chain structure, the latter $\pi$ orbitals form a doubly degenerate but half-filled band, which cross the Fermi level. The double bond structure underlies the unusual properties of $\mathrm{C}_{n}$, such as its high axial strength, transversal flexibility, and strong cohesion. For example, the elastic stiffness of $\mathrm{C}_{n}$, i.e., the second derivative of the strain energy per atom with respect to the axial strain, $d^{2} E / d \varepsilon^{2}$, was calculated to be $119 \mathrm{eV}$, which is twice the value calculated for the carbon nanotubes. ${ }^{51}$ Despite its low coordination number of two as compared to four in diamond or three in graphite, the cohesion energy of $\mathrm{C}_{n}$ is as large as $90 \%$ of that of diamond crystal. Mechanical, electronic and magnetic properties of $\mathrm{TM}-\mathrm{C}_{n}-\mathrm{TM}$ are derived from those of carbon strings. Our concern is first to demonstrate that $\mathrm{TM}-\mathrm{C}_{n}-\mathrm{TM}$ chains are stable and that their synthesis is energetically feasible.

Transition state analysis performed for different reaction paths provides us with the conclusive evidences showing that the linear $\mathrm{TM}-\mathrm{C}_{n}-\mathrm{TM}$ atomic chains are stable and can be synthesized. In Fig. 1, we present the energetics related with the formation of $\mathrm{CoC}_{7} \mathrm{Co}$ and $\mathrm{CrC}_{7} \mathrm{Cr}$ atomic chains. These chains can conveniently grow from a finite $\mathrm{C}_{n}$ chain by attaching TM atoms from both ends. No energy barrier is involved in the course of the binding. First, a single $\mathrm{Cr}$ atom is attached to the left end of the chain consisting of seven 

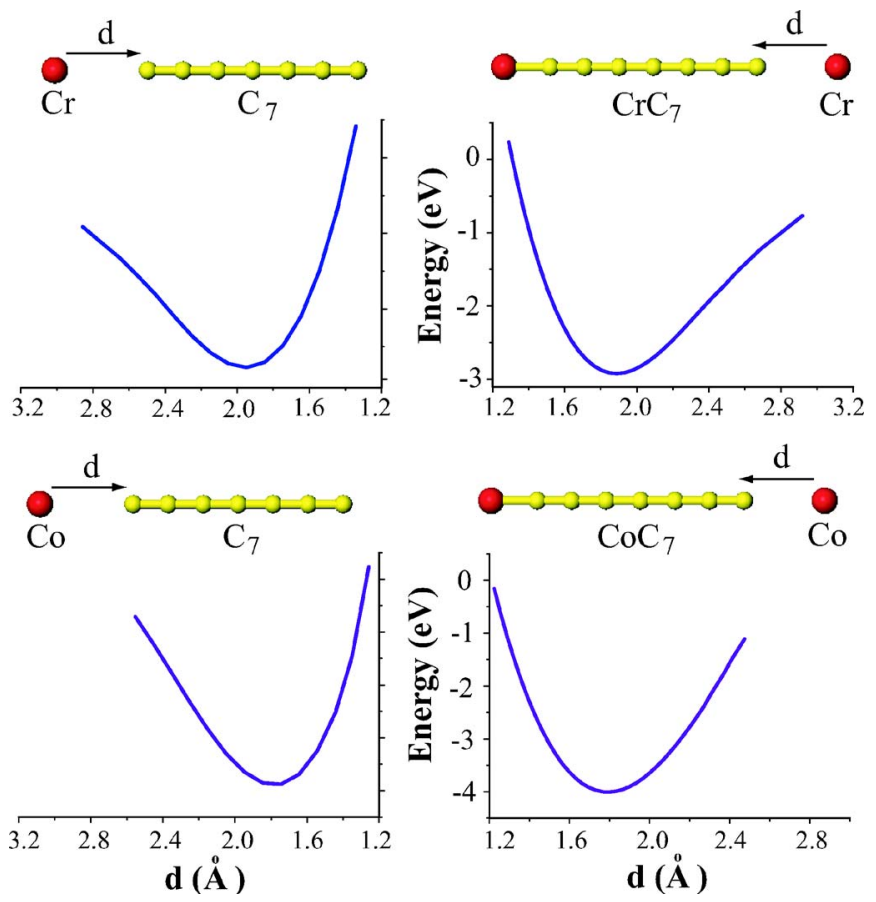

FIG. 1. (Color online) Energetics of the formation of $\mathrm{CoC}_{7} \mathrm{Co}$ and $\mathrm{CrC}_{7} \mathrm{Cr}$ atomic chains. Left panels correspond to a $\mathrm{TM}$ atom attaching to the left free end of the bare carbon chain; right panels are for the binding of a TM atom to the other end of the TM-C chain. $d$ is the distance between TM atom and $\mathrm{C}$ atom. The total energy of the system for $d \rightarrow \infty$ is set to zero in each panel.

carbon atoms. Then, the second $\mathrm{Cr}$ atom is attached to the free right end. As expected, the variation of the binding energy, $E_{b}$, of the $\mathrm{Cr}$ atom with respect to its distance $d$ from the end of the carbon chain is similar for both $\mathrm{Cr}$ atoms. The symmetry of the $E_{b}(d)$ curves for the left and right panels in Fig. 1 can be broken for small $n$, where significant direct coupling between two TM atoms can be present. The binding energy of the Co atoms are found to be larger than that of $\mathrm{Cr}$. The binding energies in the range of $2-4 \mathrm{eV} /$ per TM atom indicate an exothermic process. The energetics of growth clearly demonstrates that TM- $\mathrm{C}_{n}$-TM chains are not simply a theoretical construct of fundamental interest, but they can also be realized experimentally. To this end, carbon monatomic chain produced at the center of a multiwall carbon nanotube,${ }^{54}$ can be used as the initial stage of the fabrication. This way carbon nanotube itself encapsulates the compound and protects it from oxidation and chemisorption of foreign atoms. Experiments can be achieved by atomic manipulation using atomic force microscopy.

The optimized atomic structure of TM- $\mathrm{C}_{n}-\mathrm{TM}$ atomic chains, in particular interatomic distances slightly vary depending on whether the chain is in ferromagnetic or antiferromagnetic state. However, a systematic variation based on the type of the magnetic state could not be deduced. On the other hand, in Fig. 2 it is seen that $\mathrm{C}-\mathrm{C}$ bond lengths of the chains exhibit a significant difference depending on $n$ being odd or even. We note that $\mathrm{C}-\mathrm{C}$ double bonds are rather uniform with $d \sim 1.28 \AA$ when the number of carbon atoms $n$ is odd for both the FM and AF states. However, in the cases

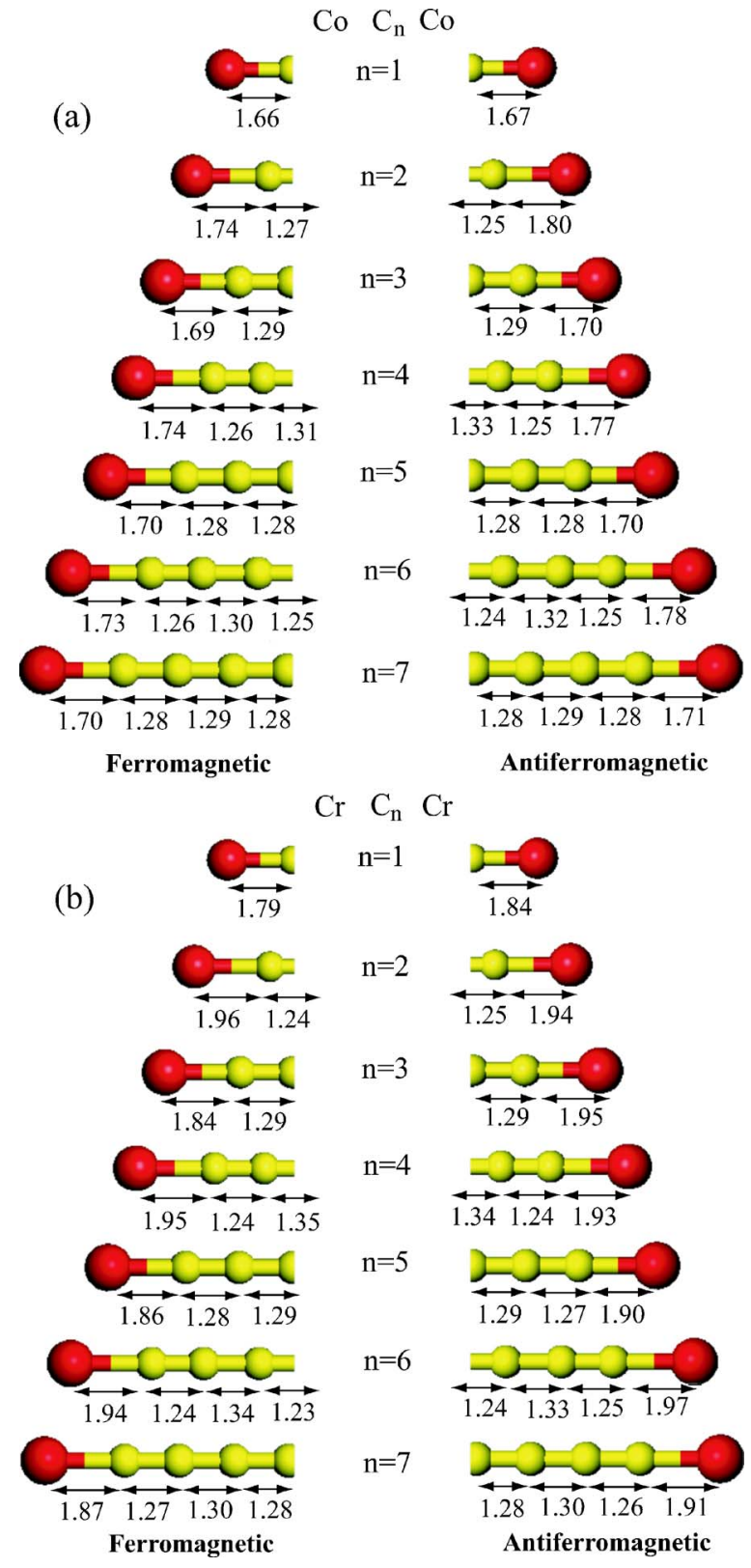

FIG. 2. (Color online) Optimized interatomic distances (in $\AA$ ) of

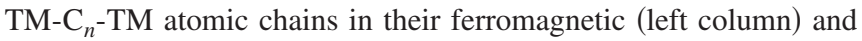
antiferromagnetic (right column) states. (a) $\mathrm{CoC}_{n} \mathrm{Co}$; (b) $\mathrm{CrC}_{n} \mathrm{Cr}$.

with even $n$ there exists a bond-length alternation with two different types of C-C bonds. For the even $n$ case, alternating single and triple $\mathrm{C}-\mathrm{C}$ bonds with $d_{\sin } \sim 1.33 \AA$ and $d_{\text {tri }} \sim 1.25 \AA$ are realized. When the carbon chain tends to dimerize the terminal $\mathrm{Cr}$ or $\mathrm{Co}$ atoms have longer bond lengths. The even-odd disparity displayed by the length and hence the types of bonds originate from the symmetry.

\section{B. Ab initio molecular dynamics calculations}

It might be expected that the chain structures are vulnerable to clustering due to random motion of individual atoms at elevated temperatures, if the equilibrium structure has instabilities. To check this effect we further tested the stability 


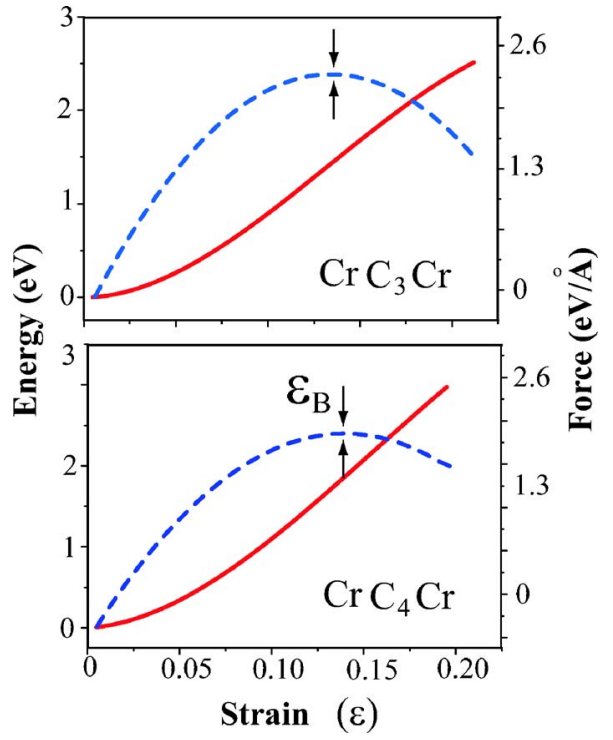

FIG. 3. (Color online) Optimized total energy (continuous line) and tensile force (dashed line) vs strain of $\mathrm{CrC}_{3} \mathrm{Cr}$ and $\mathrm{CrC}_{4} \mathrm{Cr}$ atomic chains. The chain breaks for strain values exceeding the critical point corresponding to the maximum of the force curve indicated by arrows. The total energies in equilibrium are set to zero.

of the $\mathrm{TM}-\mathrm{C}_{n}-\mathrm{TM}$ linear chains by carrying out $a b$ initio molecular dynamics calculations at high temperatures using Langevin thermostat. ${ }^{46,55}$ Calculations carried out for all structures at high temperatures $(800 \mathrm{~K}<T<1200 \mathrm{~K})$, for 250 time steps $(0.5 \mathrm{ps})$ confirmed the stability of linear chain geometry.

\section{Breaking strength of the atomic chains}

A crucial property of atomic chains is their stability against applied axial stress. The breaking strength is the maximum strain that a $\mathrm{TM}-\mathrm{C}_{n}-\mathrm{TM}$ atomic chain can sustain. Variations of total energy and the tensile force as a function of $\varepsilon$ in $\mathrm{CrC}_{n} \mathrm{Cr}$ for $n=3$ and 4 are presented in Fig. 3. Here the tensile strain is defined as the fractional elongation of the chain, namely $\varepsilon=\left(L-L_{0}\right) / L_{0}$, where $L_{0}$ is the equilibrium length of the chain, and $L$ is the length of the structure under the applied tensile force $F_{t}$. We note that $F_{t}=-L_{0}^{-1} \partial E_{T} / \partial \varepsilon$. For small tensile strain $(\varepsilon>0)$ the variation of $E_{T}$ is parabolic. Initially, the tensile force, $F_{t}$ increases with increasing $\varepsilon$, passes through a maximum that corresponds to an inflection point of attractive $E_{T}$. Further increase of $\varepsilon$ leads to decreasing of $F_{t}$. The maximum of $F_{t}$ corresponds to the breaking point of the chain. Since the carbon-carbon bonds are much stronger, the chain breaks at one of the $\mathrm{Cr}$ contacts. In Fig. 3 the breaking strain is estimated to be $\varepsilon_{B} \sim 13 \%$.

\section{MAGNETIC PROPERTIES}

The principle character of the $\mathrm{TM}-\mathrm{C}_{n}-\mathrm{TM}$ atomic chains is their magnetic ground state that varies with $n$. The exchange interaction of the magnetic TM atoms through the nonmagnetic carbon chain determines the magnetic ordering in these molecular structures. In order to find the ground state magnetic moment, the total energy of the TM-C ${ }_{n}-\mathrm{TM}$ structure is calculated for each possible value of its magnetic moment, since spin-relaxed calculations may sometimes fail to reach the lowest-energy magnetic state within the numerical algorithms available. If AF state is not the ground state, it is in general lowest-energy configuration of a FM ground state in the TM- $\mathrm{C}_{n}-\mathrm{TM}$ structures. The energy difference of the AF and the lowest-energy FM states, $\Delta E_{F M \rightarrow A F}=E_{T}(A F)-E_{T}(F M)$, is a measure of the strength of the exchange interaction between the two TM atoms, and it is tabulated in Table I for all the elements of the $3 d$ TM row of the periodic table, with $n=1$ to 7 . A negative value of $\Delta E_{F M \rightarrow A F}$ corresponds to an AF ground state. It is the energy required to invert the local magnetic moment on one of the TM atoms to obtain an antiparallel alignment of the moments on the TM atoms starting from the parallel alignment (FM state). The net molecular magnetic moments corresponding to the ground state are also tabulated in Table I (if the ground state is AF with $\mu=0$, the moment of the higher energy FM state is given in parenthesis). The total energy calculations performed using spin polarized local-density-approximation $(\mathrm{LDA})^{55}$ resulted in energies similar to those obtained by using spin-polarized GGA. For example, $\Delta E_{F M \rightarrow A F}$ of $\mathrm{CrC}_{3} \mathrm{Cr}$ is calculated to be $1.03 \mathrm{eV}$ and $0.87 \mathrm{eV}$ using LDA and GGA, respectively. Similarly $\mathrm{CrC}_{4} \mathrm{Cr}$ has $\Delta E_{F M \rightarrow A F}=$ -0.11 and $-0.08 \mathrm{eV}$ by using LDA and GGA, respectively. In particular, the magnetic order in the ground states of chain structures are found to be robust and does not change when either one switches from GGA or LDA are employed.

An interesting feature revealed from the Table $\mathrm{I}$ is for a given TM atom the ground state of the $\mathrm{TM}-\mathrm{C}_{n}-\mathrm{TM}$ chain varies between FM and AF configurations as a function of the number of carbon atoms. The variations are dominantly in the form of regular alternations with a period of two atoms in particular for $\mathrm{V}, \mathrm{Cr}, \mathrm{Fe}$, and $\mathrm{Co}$. For the $\mathrm{CrC}_{n} \mathrm{Cr}$ molecules, the ground states are AF for even $n$, where the first excited state is FM with a total magnetic moment $\mu=10 \mu_{B}$ ( $\mu_{B}$ is the Bohr magneton). However for odd $n$ the ground state is $\mathrm{FM}$ with $\mu=8 \mu_{B}$. Calculations for $\mathrm{CoC}_{n} \mathrm{Co}$ molecules show a similar but inverted behavior. The ground state is $\mathrm{AF}$ for odd $n$ and the energy difference $\Delta E_{F M \rightarrow A F}$ again oscillates in sign with the variation of $n$ but the signs are inverted. This even-odd $n$ alternation is inherent to atomic chain structures, and manifests itself in the electronic and conductance properties of atomic chains. ${ }^{51,56,57}$ The regular alternation of the magnetic ground state for longer $\mathrm{CrC}_{n} \mathrm{Cr}$ and $\mathrm{CoC}_{n} \mathrm{Co}$ chains up to $n=15$ have been found to persist.

The strength of the exchange interaction decays slowly with increasing $n$, as expected. In Fig. 4 we consider the case for $\mathrm{CrC}_{n} \mathrm{Cr}$ as an example. The decay rate of the interaction as a function of the distance $d$ between the $\mathrm{Cr}$ atoms is empirically deduced by fitting the $\Delta E_{F M \rightarrow A F}$ values to a simple power dependence $\sim d^{\alpha}$. The value of $\alpha$ turns out to be -0.72 and -1.43 for the FM and AF configurations, respectively. Models for extended systems [such as Ruderman-KittelKasuya-Yosida (RKKY)] that describe the exchange interaction of magnetic moments embedded in nonmagnetic media predict an asymptotic decay of the interaction in the form $d^{-1}$ in one-dimensional systems. As will be discussed later in this 
TABLE I. The energy difference of the AF and the lowest-energy FM states, $\Delta E_{F M \rightarrow A F}=E_{T}(A F)$ $-E_{T}(F M)$ in $\mathrm{eV}$, and the magnetic moment $\mu$ of the ground state in units of Bohr magneton $\mu_{B}$. For cases with $\mathrm{AF}$ ground states the moment corresponding to the lowest-energy FM state is given in parenthesis.

\begin{tabular}{lccccccc}
\hline \hline \multirow{2}{*}{$\mathrm{TM}-\mathrm{C}_{n}$ - TM } & \multicolumn{7}{c}{ Number of C atoms, $n$} \\
$\mathrm{TM}$ & 1 & 2 & 3 & 4 & 5 & 6 & 7 \\
\hline $\mathrm{Sc}$ & -0.06 & -0.11 & -0.32 & -0.10 & -0.02 & -0.06 & -0.02 \\
& $(2)$ & $(2)$ & $(2)$ & $(4)$ & $(2)$ & $(4)$ & $(2)$ \\
$\mathrm{Ti}$ & -0.28 & 0.41 & 0.36 & 0.40 & -0.16 & 0.10 & -0.16 \\
& $(4)$ & 6 & 4 & 6 & $(4)$ & 6 & $(4)$ \\
$\mathrm{V}$ & -0.27 & 0.48 & -0.34 & 0.37 & -0.39 & 0.31 & -0.27 \\
& $(6)$ & 8 & $(6)$ & 8 & $(6)$ & 8 & $(6)$ \\
$\mathrm{Cr}$ & 1.12 & -0.10 & 0.87 & -0.08 & 0.70 & -0.06 & 0.58 \\
& 8 & $(10)$ & 8 & $(10)$ & 8 & $(10)$ & 8 \\
$\mathrm{Mn}$ & -0.09 & -0.07 & 0.24 & -0.04 & 0.29 & -0.03 & 0.20 \\
& $(10)$ & $(12)$ & 10 & $(12)$ & 10 & $(12)$ & 10 \\
$\mathrm{Fe}$ & -0.34 & 0.34 & -0.33 & 0.23 & -0.32 & 0.19 & -0.31 \\
& $(2)$ & 6 & $(4)$ & 6 & $(4)$ & 6 & $(4)$ \\
$\mathrm{Co}$ & -0.17 & 0.32 & -0.12 & 0.28 & -0.13 & 0.24 & -0.13 \\
& $(2)$ & 4 & $(2)$ & 4 & $(2)$ & 4 & $(2)$ \\
$\mathrm{Ni}$ & 0.00 & 1.18 & 0.00 & 0.18 & 0.00 & 0.07 & 0.00 \\
& 0 & 2 & 0 & 2 & 0 & 2 & 0 \\
\hline \hline
\end{tabular}

section, this discrepancy exemplifies that conventional models are not readily applicable to the present molecular TM- $\mathrm{C}_{n}$-TM structures.

The $3 d \mathrm{TM}$ atoms can be grouped into sets according to the $n$-dependent ground state variations of the $\mathrm{TM}-\mathrm{C}_{n}$-TM chains. Namely, Ti, V, and Fe display Co-like regular alternations, with the exception of $\mathrm{TiC}_{3} \mathrm{Ti}$, where the ground state is found to be FM, although all other odd- $n$ cases have $\mathrm{AF}$ states. $\mathrm{Mn}, \mathrm{Cr}$, and also Mo from the $4 d$ row, on the other hand, display an inverted alternation where $\mathrm{FM}$ and $\mathrm{AF}$ states are interchanged relative to the first set of elements. Again, $\mathrm{MnC}_{1} \mathrm{Mn}$ is an exception. The latter set of $\mathrm{TM}$ atoms have the common property of half-filled $d$ shells, namely the electronic configurations of the neutral atoms having $3 d^{5}$. The variations of the magnetic ground state with $n$ of the

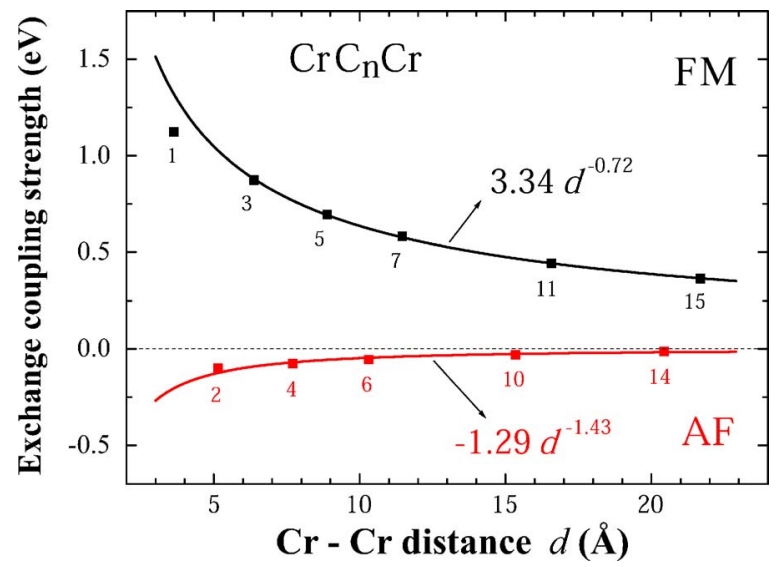

FIG. 4. (Color online) The decay of the exchange interaction strength between the $\mathrm{Cr}$ atoms in $\mathrm{CrC}_{n} \mathrm{Cr}$ as a function of their separation $d$. The curves are the best fits to the $\Delta E_{F M \rightarrow A F}$ values in the form $\sim d^{\alpha}$. chains made by $\mathrm{Sc}$ and $\mathrm{Ni}$ atoms, the far-end elements of the $3 d$ row of periodic table, are different from the others. For Sc, all cases with $n=1$ to 7 have AF ground states. Although the behavior of $\mathrm{Ni}$ resembles to that of the Co group in that the even- $n$ cases have FM ground states, the odd- $n$ cases are nonmagnetic with $\mu=0$. Figure 5 illustrates the variations of $\Delta E_{F M \rightarrow A F}$ versus the number $n$ of carbon atoms in the chain for the three different sets of TM atoms.

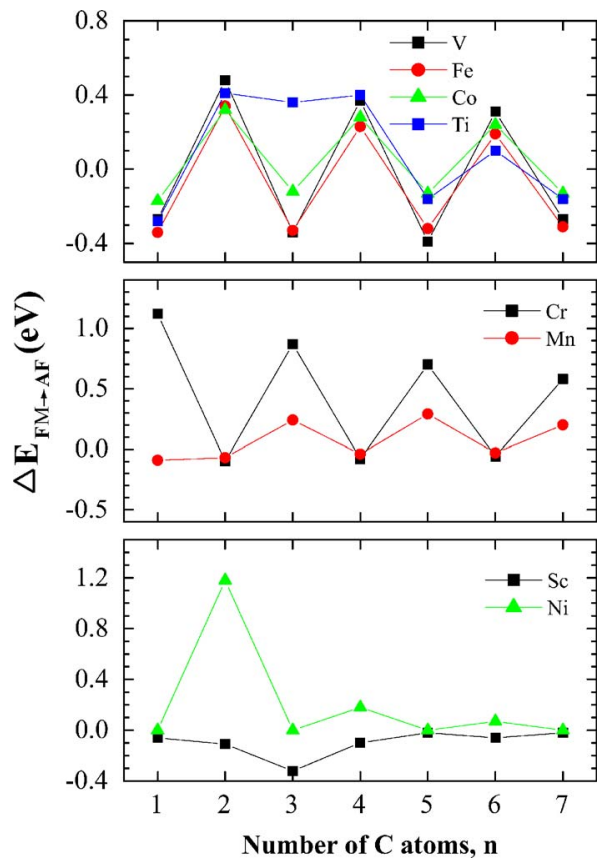

FIG. 5. (Color online) The energy difference of the AF and the lowest-energy FM states, $\Delta E_{F M \rightarrow A F}=E_{T}(A F)-E_{T}(F M)$ vs the number $n$ of carbon atoms in the chain for different $3 d$ transition metal elements. 

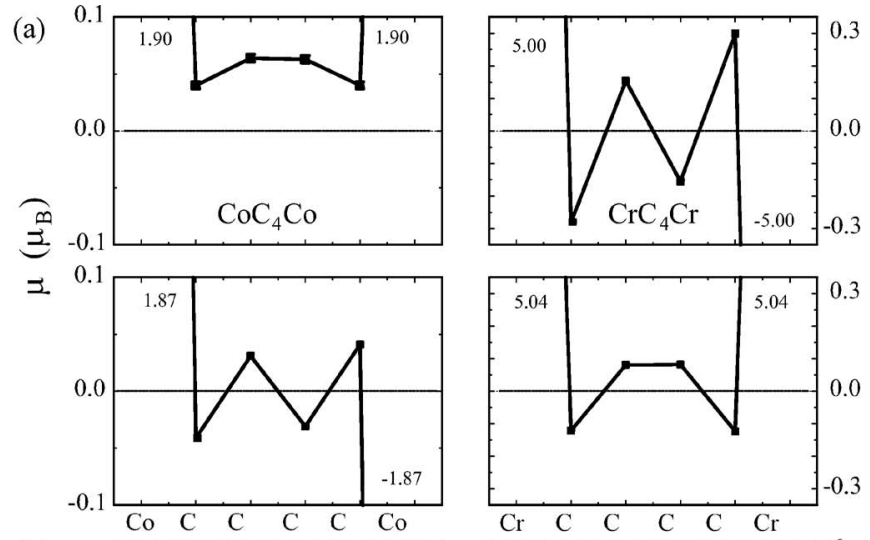

(b)
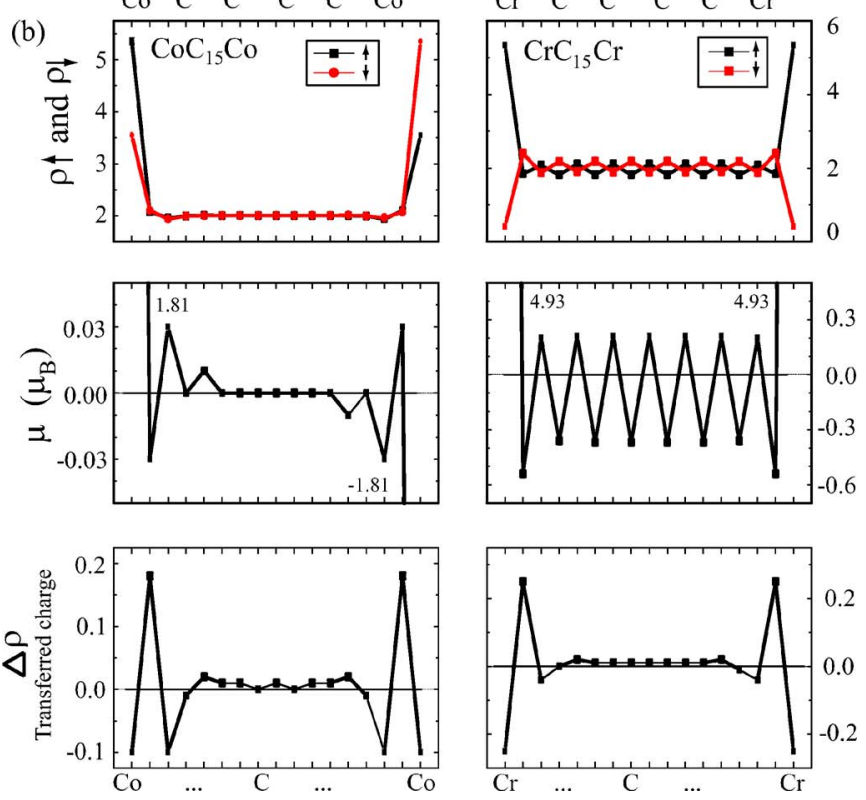

FIG. 6. (Color online) (a) Variation of the atomic magnetic moments in the $\mathrm{TM}^{-\mathrm{C}_{4}}$ - TM atomic chains in their ground (top panels) and excited (down panels) states. Left (right) panels are for $\mathrm{TM}=\mathrm{Co}(\mathrm{TM}=\mathrm{Cr})$. (b) From top to bottom: Variation of spin-up $\rho \uparrow$ and spin-down charge $\rho \downarrow$ densities, atomic magnetic moments, and change in total valance charge $\Delta \rho$ in the $\mathrm{TM}-\mathrm{C}_{15}-\mathrm{TM}$ atomic chains. Again, left (right) panels are for $\mathrm{TM}=\mathrm{Co}(\mathrm{TM}=\mathrm{Cr})$.

In the $\mathrm{TM}-\mathrm{C}_{n}-\mathrm{TM}$ molecules not only the magnetic ground state and the total molecular magnetic moment but also the distribution of the atomic magnetic moments display interesting variations. The spin-dependent interactions within the molecule create distortions in the spin populations of the carbon atoms, leading to induced magnetic moments on the carbons too, which are nonmagnetic otherwise. We calculate the atomic magnetic moments based on an orbital-resolved Mulliken analysis. ${ }^{47}$ In Fig. 6(a) $\mathrm{CoC}_{4} \mathrm{Co}$ and $\mathrm{CrC}_{4} \mathrm{Cr}$ are considered as sample cases in their ground and first excited magnetic states. Several distinct forms of atomic magnetic moment distribution on the carbon chain are obtained depending on the magnetic state of the molecule, type of the TM atom, and the length of the chain. The induced magnetic moments on the carbon atoms neighboring to the $\mathrm{Cr}$ atoms are as large as $|\mu| \sim 0.3 \mu_{B}$ in $\mathrm{CrC}_{4} \mathrm{Cr}$. Owing to perfect linear geometry and quantum interference effects the induced magnetization of $\mathrm{C}$ atoms can be long ranged. In Fig. 6(b) we display their variation in the ground states of two longer molecules, $\mathrm{CoC}_{15} \mathrm{Co}$ and $\mathrm{CrC}_{15} \mathrm{Cr}$, together with atomic spin populations and changes in total valance charges relative to isolated atoms. We observe that in the FM ground state of $\mathrm{CrC}_{15} \mathrm{Cr}$, even though the total charge transfer of the carbon atoms are small except for the end atoms, there induces considerable spin imbalance on the carbon atoms leading to atomic magnetic moments alternating in sign.

The mechanism of the long-range exchange interaction between the TM atoms in the TM- $\mathrm{C}_{n}-\mathrm{TM}$ structures can be inferred from the analysis of spin dependent interactions. It is in some respects reminiscent of RKKY interaction ${ }^{58}$ which deals with the coupling between magnetic impurities in a nonmagnetic host, and the interlayer exchange coupling of magnetic layers separated by nonmagnetic spacer layers. ${ }^{59-61}$ RKKY interaction is a second-order perturbative effect that plays a significant role in determining the coupling of localized $d$-shell electron spins in a metal by means of an interaction through the conduction electrons of the medium. The ferromagnetic and/or antiferromagnetic oscillations in the indirect exchange coupling of the magnetic impurities is a prediction of the RKKY theory. The interlayer exchange coupling theories explain the oscillatory variations in terms of the spin dependent change of the density of states due to quantum interferences generated by multiple reflections from the interfaces. In both formulations the oscillatory exchange coupling is related to the sharp cutoffs in momentum space due to the Fermi surfaces of the host or the spacer media.

Here, owing to the quasi-zero-dimensional nature and hence finite level spacing of the TM- $\mathrm{C}_{n}$-TM structures, and nonperturbative character of the interaction of the TM atoms with the carbon chain a different treatment is required. One needs to employ self-consistent density functional methods or a direct diagonalization of the spin-dependent model Hamiltonian of the system. In the following we present a simple model that can explain the qualitative features of the indirect exchange coupling in the $\mathrm{TM}-\mathrm{C}_{n}-\mathrm{TM}$ molecular structures.

\section{A. A tight-binding model}

Variation of the exchange coupling between magnetic layers or atoms separated by nonmagnetic spacers has been widely studied for extended bulk or layered systems both experimentally and theoretically. In our case of TM-atom capped carbon chains, the nonperiodic nature of the system, and the strength of the spin-dependent interactions requires a model which can take into account the molecular character of the system. We propose a simple model in order to explain the dominant mechanism of the exchange interaction between the TM atoms through a quasi-zero-dimensional nonmagnetic spacer, i.e., the finite carbon chain. Two main features of the interaction to be simulated within our model are (i) the variation of the energy difference between the FM and AF states of the chain (namely $\Delta E_{F M \rightarrow A F}$ ) with respect to the number of carbon atoms present, (ii) the dependence of atomic magnetic moments on the number of $\mathrm{C}$ atoms, and on the species of the TM atom. We keep the model as simple as possible for the clarity of the basic mechanism. 


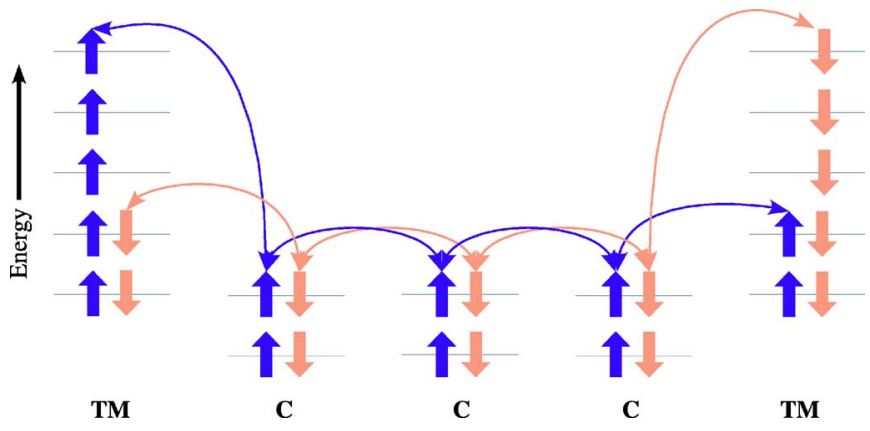

FIG. 7. (Color online) Schematic electronic configuration of a TM- $\mathrm{C}_{n}$-TM structure in an AF state. TM sites have different on-site energies than the $\mathrm{C}$ sites, and the hopping terms are dependent on the magnetic ordering. The energy cost for a spin-up electron of the first $\mathrm{C}$ site to hop to the first TM site is different from the energy cost for a spin-down electron to do the same hopping. The energy costs are reversed between the spins of the $n$th $\mathrm{C}$ site where they are identical for each spin for hopping between different $\mathrm{C}$ sites.

Consider a tight-binding model Hamiltonian where each atom is represented by a site with a single level per spin type. We allow only the first nearest-neighbor hopping.

$$
H=\sum_{i=0 ; \sigma}^{n+1} \epsilon_{i, \sigma} c_{i, \sigma}^{+} c_{i, \sigma}-\sum_{i=0 ; \sigma}^{n} t_{i, i+1 ; \sigma} c_{i, \sigma}^{+} c_{i+1, \sigma}+\text { H.c. }
$$

where $i$ is the site index. Zeroth and $(n+1)$ th sites are the TM sites, in between are the carbon sites. $c_{i, \sigma}$ and $c_{i, \sigma}^{+}$are the annihilation and creation operators for an electron with spin $\sigma(\sigma=\uparrow, \downarrow)$. The onsite energies $\epsilon_{i, \sigma}$ and the hopping terms $t_{i, i+1 ; \sigma}$ are both spin dependent. The nonmagnetic carbon sites are represented by spin-degenerate parameters, and the effect of TM capping is simulated by assigning spin-dependent on site and coupling parameters to the TM sites.

The relative strengths of the spin-dependent parameters of the TM sites can be inferred from the electronic structure of isolated TM atoms. The number of spin-up and spin-down electrons are different for a TM atom and the highest occupied (lowest unoccupied) spin up atomic level is different from that of the down spin. This enters to our model as different on-site parameters for each spin. The effective coupling parameters of the two spin states to the neighboring $\mathrm{C}$ sites will be different for the same reason. We choose the magnetic moment of the left TM site as positive and that of the right TM site is to be chosen with respect to the magnetic state of the molecule, that is positive for ferromagnetic and negative for antiferromagnetic alignments. In Fig. 7 a schematic plot of valence electron distributions that can be correlated to the relative strengths of the model Hamiltonian parameters is shown.

An interpretation of this model is possible if one makes an analogy with a particle in a one dimensional potential well. ${ }^{61}$ One needs to consider two different potential profiles for the electrons of each spin type. The potential for the majority spin electrons at the left TM site is higher than the potential at the spacer, namely, the well region. It leads to symmetric potential profiles for each spin type for the ferromagnetic configuration and antisymmetric ones for the antiferromagnetic case.

The Hamiltonian is then characterized in terms of parameters $\left\{E_{1}, E_{2}, t_{1}, t_{2}\right\}$ where $E_{1}=\epsilon_{0 ; \uparrow}, E_{2}=\epsilon_{0 ; \downarrow}$ are the on-site energies for the majority and minority spins of the left TM site and $t_{1}=t_{0,1 ; \uparrow}, t_{2}=t_{0,1 ; \downarrow}$ are their coupling energies to the nearest carbon site. We set the onsite energy of the carbon sites to zero as reference and C-C hopping parameter to $t$ for both spin types. As we consider the same species of TM atoms at both ends, the on-site and hopping parameters of the right TM site are chosen in accordance with the particular magnetic order of the molecule (FM or AF). Having written each parameter in units of $t$, we diagonalize spin-up and spin-down Hamiltonians of the system separately since we do not consider any spin-flip interactions. The energy spectrum for each spin type in both FM and AF states of the molecule is calculated, and half filling is applied to the combined spectra to end up with the total energies of the FM and AF configurations.

We consider $\mathrm{Co}$ and $\mathrm{Cr}$ as the cap TM atoms. Isolated $\mathrm{Cr}$ has five majority and zero minority spins in its $d$ shell, whereas isolated Co has five majority and two minority spins. When the TM atom is chemically bound to the $\mathrm{C}$ chain from left, the electrons of the leftmost $\mathrm{C}$ atom will experience different interaction potentials depending on their spins, and the coupling terms to the TM site will also be spin dependent.

The energy cost for a majority spin electron to hop from the $\mathrm{C}$ site to the TM site is expected to be comparable for both $\mathrm{Co}$ and $\mathrm{Cr}$ atoms in view of their isolated electronic configuration. On the other hand, the energy required for a minority spin electron to hop from the $\mathrm{C}$ site to the TM site should be larger for Co atom than it is for Cr atom. Similarly, the hopping terms are different for minority spin electrons hopping to $\mathrm{Co}$ or $\mathrm{Cr}$ atoms. Along these arguments, we find that the parameter sets $\{1.0,0.0,1.5,1.0\}$ and $\{1.0,0.3,1.5$, $0.5\}$ for $\mathrm{Cr}$ and $\mathrm{Co}$, respectively, lead to results in fairly good qualitative agreement with our first-principles DFT results. The difference in total energy of the molecule for varying number of $\mathrm{C}$ atoms is presented in Fig. 8, as calculated using both the DFT methods and the simple tight-binding model. Although the model does not include any self-consistent calculations for the electronic configuration it is capable of representing the basic physical mechanism underlying the magnetic state dependence of the TM-capped $\mathrm{C}$ chains on the number of atoms in the $\mathrm{C}$ chain.

\section{B. Asymmetric atomic chains}

So far we have discussed the symmetric $\mathrm{TM}^{-\mathrm{C}_{n}}$-TM finite chain structures. How the above features change when the atomic symmetry of the chain is broken or the chain has only one TM atom like $\mathrm{C}_{n}-\mathrm{TM}-\mathrm{C}_{m}$ is the question we address next. First we consider the TM- $\mathrm{C}_{n}-\mathrm{TM}$ structure having asymmetry in the type of TM, like $\mathrm{CoC}_{n} \mathrm{Cr}$ chain with $n=1-4$. As expected, $\Delta E_{F M \rightarrow A F}=E_{A F}-E_{F M}$ is large and approximately equal to $1.6 \mathrm{eV}$ for even $n$, but is relatively small and equal to $0.7 \mathrm{eV}$ for odd $n$. Nevertheless, $\Delta E_{F M \rightarrow A F}>0$ indicating that the ground state of all these 


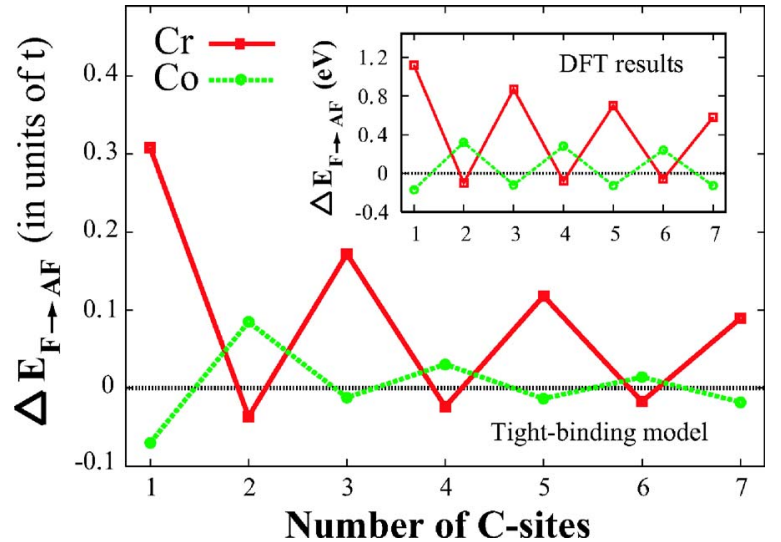

FIG. 8. (Color online) Energy difference of the AF and the lowest $\mathrm{FM}$ states in the $\mathrm{CoC}_{n} \mathrm{Co}$ and $\mathrm{CrC}_{n} \mathrm{Cr}$ atomic chains within the simple tight-binding model. The corresponding DFT results are shown in the inset for comparison.

chains is ferromagnetic for all $n ; \mu=7 \mu_{B}$ for even $n$, and $\mu=5-6 \mu_{B}$ for odd $n$.

Another interesting situation combines two chains, $\mathrm{CoC}_{n} \mathrm{Co}$ and $\mathrm{CoC}_{m}$ Co with $n=m-1$ or $n=m$, into a chain $\mathrm{CoC}_{n} \mathrm{CoC}_{m} \mathrm{Co}$. It is interesting to know the type of ground state of the chain, since $\mathrm{CoC}_{n} \mathrm{Co}$ is ferromagnetic for even $n$, but antiferromagnetic for odd $n$. We found that the chain with $n=3$ and $m=2$ is FM. It has $\Delta E_{F M \rightarrow A F}=0.32 \mathrm{eV}$ and $\mu=5 \mu_{B}$. The case $n=m=3$ has still FM ground state with $\Delta E_{F M \rightarrow A F}=0.23 \mathrm{eV}$ and $\mu=10 \mu_{B}$ in spite of the fact that $\mathrm{CoC}_{3} \mathrm{Co}$ has an $\mathrm{AF}$ ground state

Finally, simple transition metal atom in a chain, namely $\mathrm{C}_{n} \mathrm{CrC}_{m}$ can be viewed as an impurity. We considered $n=m=1-3 \quad$ symmetric case and $n=1, \quad m=2-4 ; n=2$, $m=3,4$ asymmetric cases, as shown in Fig. 9. In these chains even-odd disparity or dependence of bonding patterns on the number of $\mathrm{C}$ atoms is not observed. What we find is strong interaction between $\mathrm{Cr}$ and the nearest $\mathrm{C}$ atoms, and ferro-

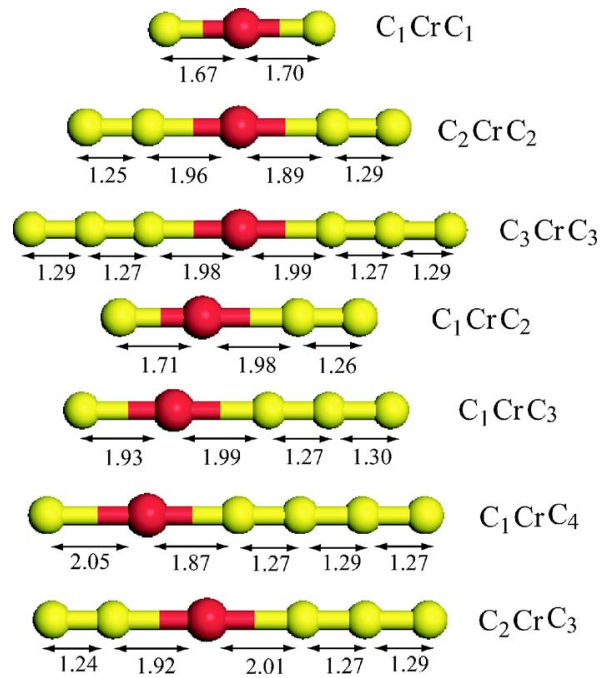

FIG. 9. (Color online) Optimized interatomic distances (in $\AA$ ) of $\mathrm{C}_{n} \mathrm{CrC}_{m}$ atomic chains in their ferromagnetic state.

magnetic ground state with $\mu=4-6 \mu_{B}$. In all cases, expect for $n=m=3$, HOMO is a spin up state. For the wires with $m>n+1$ highest occupied molecular orbital (HOMO) and lowest unoccupied molecular orbital (LUMO) become spin down states and $\mu=6 \mu_{B}$.

\section{ELECTRONIC PROPERTIES}

TM- $C_{n}$-TM chains have electronic energy structure with finite level spacing. Because of their magnetic ground state we define energy gaps separately for the minority and majority spin states. Namely, the gap for majority spin states is $E_{g}^{\uparrow}=E_{L U M O}^{\uparrow}-E_{\text {HОMO }}^{\uparrow}$. Similarly, for the minority spin states the energy gap is $E_{g}^{\downarrow}=E_{L U M O}^{\downarrow}-E_{\text {НОмO }}^{\downarrow}$. In Fig. 10 we show spin dependent HOMO and LUMO energy levels of $\mathrm{CoC}_{n} \mathrm{Co}$ and $\mathrm{CrC}_{n} \mathrm{Cr}$ (for $n=1, \ldots, 7$ ) for their magnetic ground and

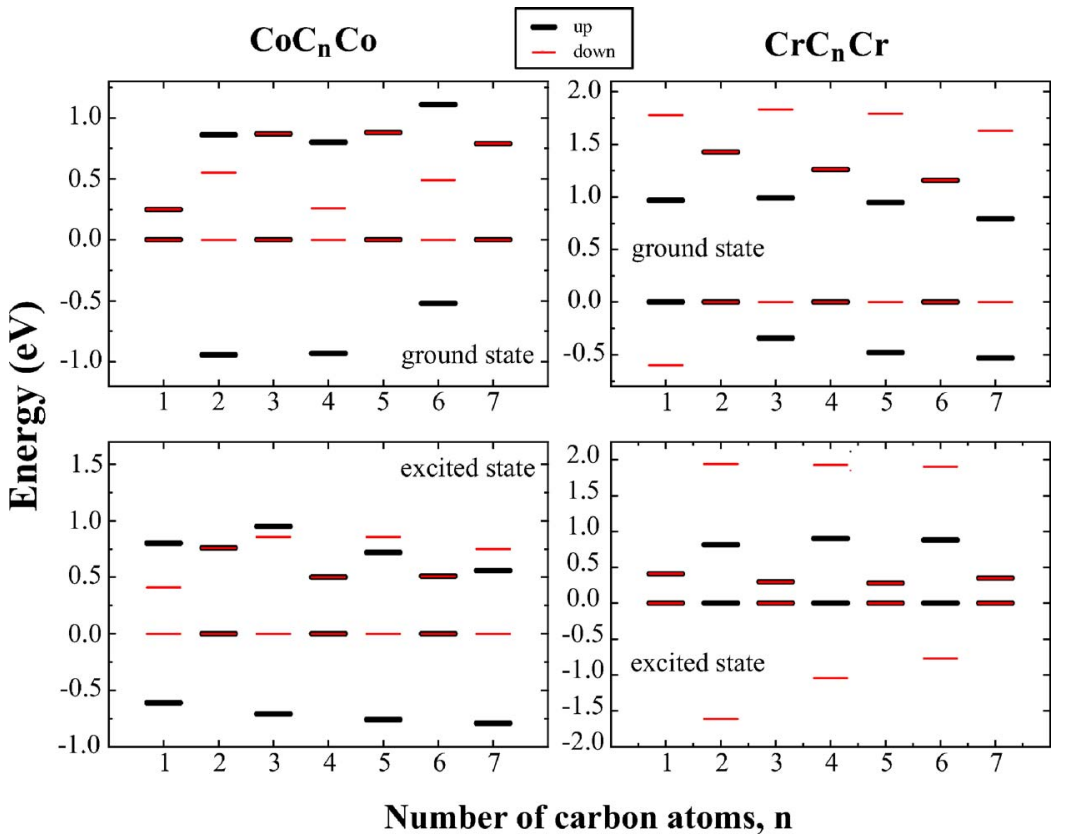

FIG. 10. (Color online) Spin dependent HOMO and LUMO levels of TM- $\mathrm{C}_{n}$-TM chains for $\mathrm{TM}=\mathrm{Co}$ and $\mathrm{Cr}, n=1-7$, in their ground and excited magnetic states. HOMO levels are set to zero in each case. 
TABLE II. Variation of electronic and magnetic properties of TM- $\mathrm{C}_{n}-\mathrm{TM}$ chains under axial strain $\varepsilon$. $\Delta E_{F M \rightarrow A F}$ is the energy difference of the AF and the lowest energy FM states given in $\mathrm{eV}$. $\mu$ is the magnetic moment in units of $\mu_{B} . E_{g}^{\sigma}$ is the energy difference in $\mathrm{eV}$ between the lowest unoccupied and highest occupied molecular orbitals for spin type $\sigma$.

\begin{tabular}{|c|c|c|c|c|c|}
\hline & $\epsilon=0$ & 0.025 & 0.050 & 0.075 & 0.100 \\
\hline \multicolumn{6}{|l|}{$\mathrm{CrC}_{3} \mathrm{Cr}$} \\
\hline$\Delta E_{F M \rightarrow A F}$ & 0.87 & 1.02 & 1.05 & 1.03 & 0.88 \\
\hline$\mu$ & 8 & 8 & 8 & 8 & 8 \\
\hline$E_{g}^{\uparrow}$ & 1.33 & 1.23 & 1.13 & 1.06 & 0.98 \\
\hline$E_{g}^{\downarrow}$ & 1.83 & 1.73 & 1.60 & 1.47 & 1.33 \\
\hline \multicolumn{6}{|l|}{$\mathrm{CrC}_{4} \mathrm{Cr}$} \\
\hline$\Delta E_{F M \rightarrow A F}$ & -0.08 & -0.07 & -0.09 & -0.10 & -0.10 \\
\hline$\mu$ & 0 & 0 & 0 & 0 & 0 \\
\hline$E_{g}^{\uparrow}$ & 1.27 & 1.13 & 0.99 & 0.85 & 0.69 \\
\hline$E_{g}^{\downarrow}$ & 1.27 & 1.13 & 0.99 & 0.85 & 0.69 \\
\hline \multicolumn{6}{|l|}{$\mathrm{CoC}_{3} \mathrm{Co}$} \\
\hline$\Delta E_{F M \rightarrow A F}$ & -0.12 & -0.14 & -0.14 & -0.14 & -0.14 \\
\hline$\mu$ & 0 & 0 & 0 & 0 & 0 \\
\hline$E_{g}^{\uparrow}$ & 0.87 & 0.87 & 0.87 & 0.74 & 0.65 \\
\hline$E_{g}^{\downarrow}$ & 0.87 & 0.87 & 0.87 & 0.74 & 0.65 \\
\hline \multicolumn{6}{|l|}{$\mathrm{CoC}_{4} \mathrm{Co}$} \\
\hline$\Delta E_{F M \rightarrow A F}$ & 0.28 & 0.15 & 0.07 & 0.04 & 0.01 \\
\hline$\mu$ & 4 & 4 & 4 & 4 & 4 \\
\hline$E_{g}^{\uparrow}$ & 1.73 & 1.60 & 1.45 & 1.29 & 1.10 \\
\hline$E_{g}^{\downarrow}$ & 0.26 & 0.48 & 0.57 & 0.62 & 0.71 \\
\hline
\end{tabular}

first excited states. $E_{g}^{\uparrow}$ and $E_{g}^{\downarrow}$ exhibit variations with $n$.

\section{A. Effect of strain}

We have shown that the $\mathrm{TM}-\mathrm{C}_{n}-\mathrm{TM}$ structures can sustain strains of $\varepsilon \sim 13 \%$ before they break. We consider how the electronic and magnetic properties of these chains change under the applied stress. We have calculated $\Delta E_{F M \rightarrow A F}, \mu$ and $E_{g}^{\uparrow}$ and $E_{g}^{\downarrow}$ as a function of $\varepsilon$. Results are listed in Table II which show that the magnetic properties are robust. The AF or FM ground state and the value of the magnetic moment $\mu$ remain unchanged. However, $E_{g}^{\uparrow}$ and $E_{g}^{\downarrow}$ display significant variation due to the relative shifts of LUMO and HOMO levels under tensile strain $0 \leqslant \varepsilon \leqslant 0.1$. In general due to the decreasing coupling between orbitals, $E_{g}^{\uparrow}$ of $\mathrm{TM}-\mathrm{C}_{n}-\mathrm{TM}$ (with $\mathrm{TM}=\mathrm{Cr}$, Co and $n=3$ and 4) decreases as $\varepsilon$ increases. This trend is inverted only for $\mathrm{CoC}_{4} \mathrm{Co}$ chain.

\section{B. Half-metallic properties}

Further, adding to the interesting magnetic and electronic properties of $\mathrm{TM}-\mathrm{C}_{n}-\mathrm{TM}$ finite size atomic chains, the periodic $\left(\mathrm{TM}-\mathrm{C}_{n}\right)_{\infty}$ chains show half-metallic properties. Half-metals (HM) are a class of materials which exhibit spin-dependent electronic properties relevant to spintronics. ${ }^{36-39}$ In HM's, due to broken spin degeneracy, energy bands $E_{n}(\mathbf{k}, \uparrow)$ and $E_{n}(\mathbf{k}, \downarrow)$ split and each band accommodates one electron per $\mathbf{k}$ point. Furthermore, they are semiconductors for one spin direction, but show metallic properties for the opposite spin direction. As a result, the difference between the number of electrons of different spin orientations in the unit cell, $N=N_{\uparrow}-N_{\downarrow}$, must be an integer and hence the spin-polarization at the Fermi level $P=\left[D\left(E_{F}, \uparrow\right)-D\left(E_{F}, \downarrow\right)\right] /\left[D\left(E_{F}, \uparrow\right)+D\left(E_{F}, \downarrow\right)\right]$ is complete. Here $D\left(E_{F}, \uparrow\right)$ is the density of states of the majority spin states. This situation is in contrast with the ferromagnetic metals, where both spin-directions contribute to the density of states at $E_{F}$ and $P$ is less than $100 \%$.

Earlier we showed that $\left(\mathrm{CrC}_{n}\right)_{\infty}(n=2, \ldots, 7)$ and $\left(\mathrm{CoC}_{n}\right)_{\infty}$ $(n=1, \ldots, 6)$ are stable periodic structures and exhibit halfmetallic properties with interesting even-odd disparities. ${ }^{40}$ Spin-dependent total density of states of $\left(\mathrm{CrC}_{n}\right)_{\infty}$ and $\left(\mathrm{CoC}_{n}\right)_{\infty}$ are presented for $n=3$ and 4 in Fig. 11. For $\left(\mathrm{CrC}_{3}\right)_{\infty}$, the majority spin bands are semiconducting with $E_{g}^{\uparrow}=0.4 \mathrm{eV}$, but the minority spin bands cross the Fermi level showing a metallic behavior. However, in $\left(\mathrm{CrC}_{4}\right)_{\infty}$ periodic chain the majority spin bands become metallic, while minority bands are semiconductor with a large gap, $E_{g}^{\downarrow}=2.9 \mathrm{eV}$. Here we note also the even-odd $n$ disparity in the spin types of metallic (semiconducting) bands. The number of carbon atoms determines whether the majority bands are an $n$-type or $p$-type semiconductor. For example, $\left(\mathrm{CrC}_{3}\right)_{\infty}$ is a $p$-type semiconductor with direct band-gap. For $\left(\mathrm{CoC}_{3}\right)_{\infty}, E_{g}^{\uparrow}$ is direct and it exhibits an $n$-type character, but it is $p$-type when $n=4$.

TM- $3 d$ orbitals play a dominant role in the electronic and magnetic properties of these periodic chains. The dispersive 


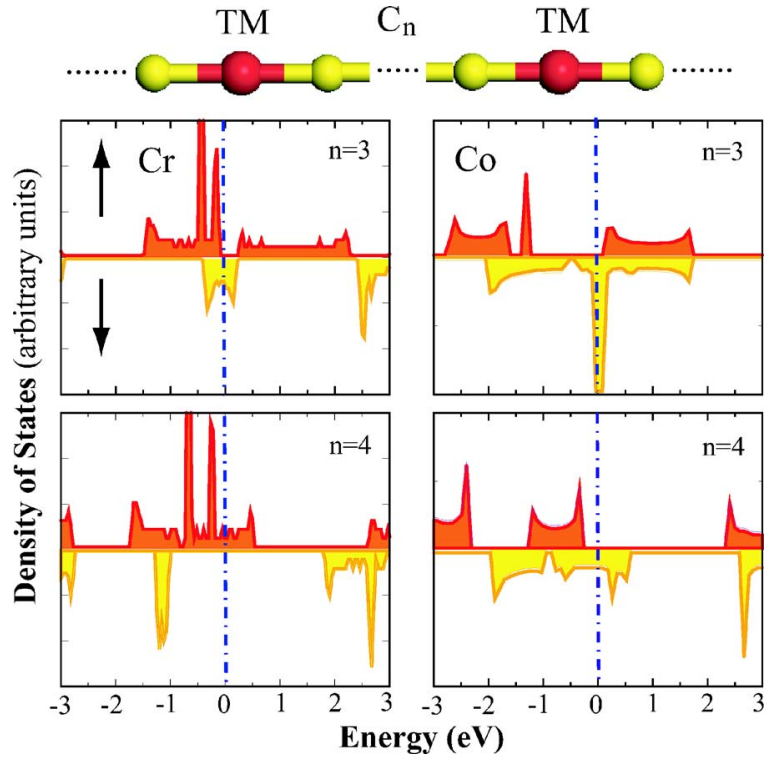

FIG. 11. (Color online) Left panels: Spin-dependent total density of states of the periodic infinite $\left(\mathrm{CrC}_{3}\right)_{\infty}$ and $\left(\mathrm{CrC}_{4}\right)_{\infty}$ atomic chains. Right panels: Same for the $\left(\mathrm{CrC}_{3}\right)_{\infty}$ and $\left(\mathrm{CrC}_{4}\right)_{\infty}$. The Fermi energy is set to zero in all systems.

bands show significant TM- $3 d$ and C- $2 p$ hybridizations. Although small modifications to the band structure can be expected due to many body effects, the band structure leading to a metallic character is found to be robust and is not affected by the axial tensile stress of $\varepsilon<0.05$. The spin-orbit coupling energy is also small and cannot influence the halfmetallicity. Calculations performed in a double unit-cell demonstrated that the Peierls instability that could have caused the splitting of the metallic bands at the Fermi level does not occur in the present systems. It should be noted that the band picture ceases in small segments of $\left(\mathrm{TM}-\mathrm{C}_{n}\right)$ due to broken translational symmetry. Then, bands are replaced by the distribution of discrete states. However, as $n$ increases the continuous state distribution of $\left(\mathrm{CoC}_{n}\right)_{\infty}$ is recovered. In summary, the indirect exchange interaction of two $\mathrm{Cr}$ (or Co) atoms in the above structures underlies the half-metallic properties.

\section{TRANSPORT PROPERTIES}

The spin-dependent properties of the isolated TM- $C_{n}$-TM chains are expected to lead magnetoresistive effects in their electronic transport properties similar to the GMR effect observed in magnetic multilayers. Only the two TM atoms play the role of the ferromagnetic layers, and the carbon chain is the spacer mediating the exchange interaction, leading to giant magnetoresistance ratios, and hence an analogous molecular scale GMR effect can be achieved.

Conductance properties of molecular devices, however, depend not only on their intrinsic structure but also on the electrodes. In particular if the coupling of the device to the electrodes is strong, the electronic structure and hence the transport properties of the device can be quite different, and cannot be inferred from the electronic structure of the isolated device. For the sake of simplicity we have considered $\mathrm{Au}$ and $\mathrm{Al}$ atomic chains to model the electrodes. Using more realistic electrodes with larger cross sections does not change the qualitative features of the magnetoresistive properties, but only increases the conductance values due to increased density of states at the electrodes. Hence, properly
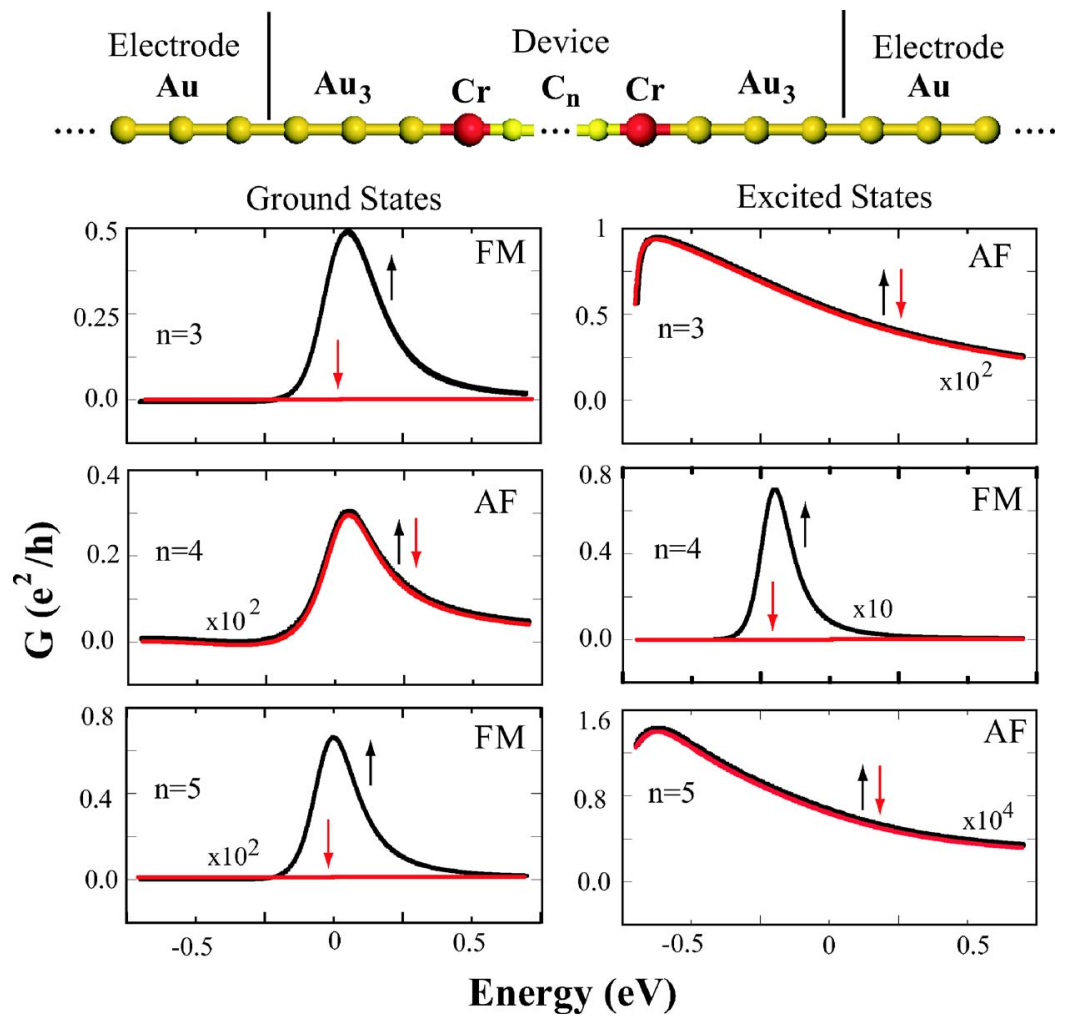

FIG. 12. (Color online) Conductance vs energy for the $\mathrm{CrC}_{n} \mathrm{Cr}(n=3-5)$ atomic chains between two infinite gold electrodes. The left (right) panels are for the ground (excited) magnetic states of the structures. The Fermi levels are set to zero. 

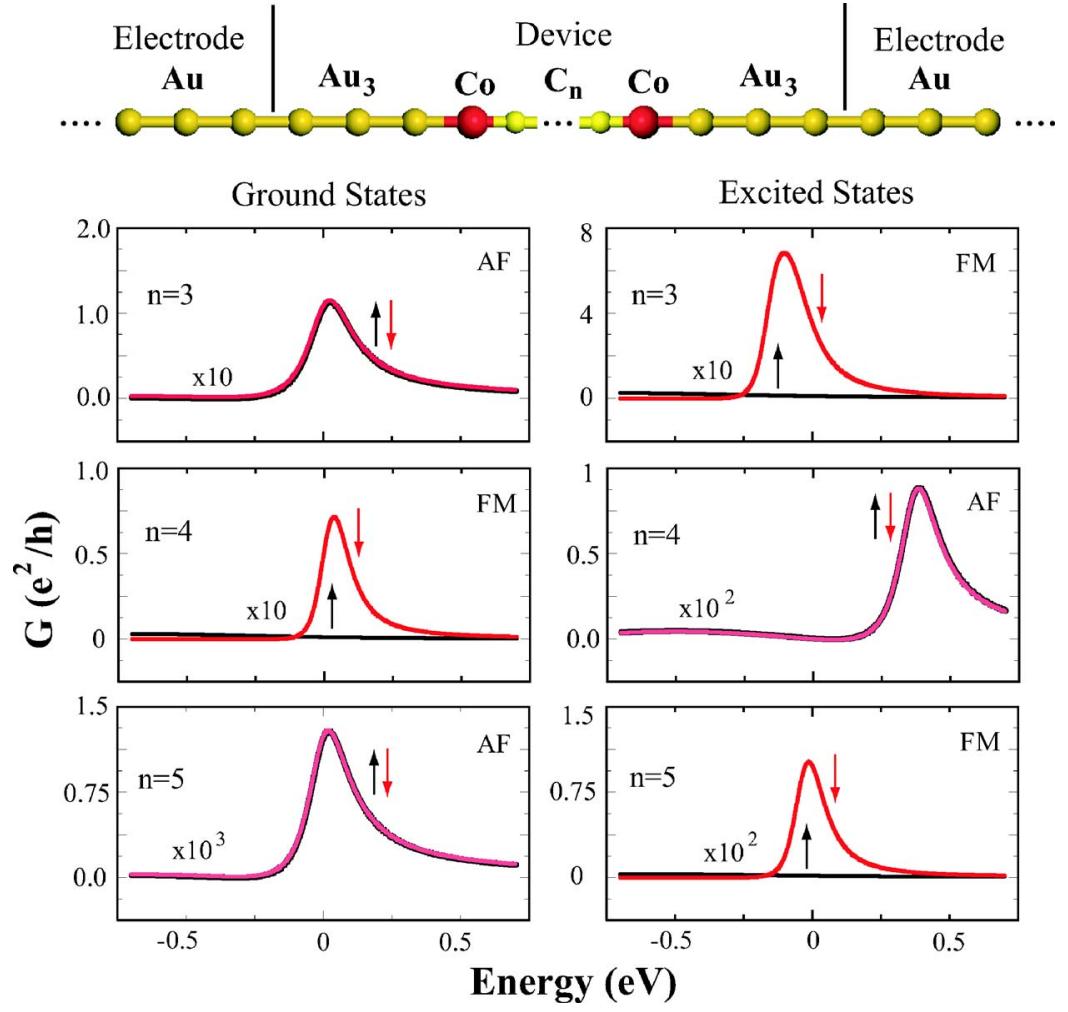

FIG. 13. (Color online) Conductance vs energy for the $\mathrm{CoC}_{n} \mathrm{Co}(n=3-5)$ atomic chains between two infinite gold electrodes. The left (right) panels are for the ground (excited) magnetic states of the structures. The Fermi levels are set to zero. treated semi-infinite atomic chain electrodes still capture the essential features of the conductance properties of these molecular-size devices. In the case of Au electrodes, we find that the AF ground state of the TM- $\mathrm{C}_{n}-\mathrm{TM}$ structures were maintained with smaller $\left|\Delta E_{F M \rightarrow A F}\right|$ values than that of their isolated forms. However, the ground states were changed to FM upon connecting to Al electrodes. In what follows we present our results obtained using Au-electrodes.

We calculate the conductance of the device within Landauer formalism, $G(E)=\left(e^{2} / h\right) \operatorname{Tr}\left(\boldsymbol{\Gamma}^{L} \mathbf{G}^{r} \boldsymbol{\Gamma}^{R} \mathbf{G}^{a}\right)$, for each spin configuration. ${ }^{49}$ In the above, $\mathbf{G}^{r}$ and $\mathbf{G}^{a}$ are retarded and advanced Green's functions, $\boldsymbol{\Gamma}^{L}$ and $\boldsymbol{\Gamma}^{R}$ are coupling functions to the left and right electrodes, respectively. In order to match the device potential and the surface potential of the semi-infinite electrodes, the device regions are defined to include some portions of the electrodes as buffer atoms. The self-consistent calculations lead to spin-up and spin-down Hamiltonians ${ }^{47}$ of the device region, which are used to calculate the transmission coefficient for each spin state in the $\mathrm{AF}$ as well as in the excited FM configuration. The surface Green's function of the contacts is calculated recursively.

Our results for the $\mathrm{CrC}_{n} \mathrm{Cr}(n=3-5)$ atomic chains, which is connected to the Au (chain) electrodes from both sides are presented in Fig. 12. The calculated conductance $G$ of these nanostructures in their ground state is given in the left panels. Since $n=3$ structure is in FM state, majority spin electrons have $G \sim 0.5 e^{2} / h$ while the minority spin electrons have negligible conductance. The situation is dramatically different for $n=4$ which has AF ground state resulting in same but relatively smaller transmission for both spin orientations. $\mathrm{CrC}_{5} \mathrm{Cr}$ has a $\mathrm{FM}$ ground state as $n=3$, but the conductance of majority spin electrons is relatively smaller due to the position of energy levels with respect to the Fermi level.
In the right panels, the transport characteristics of the excited states undergo dramatic changes. The conductance of spin-up electrons coincides with that of the spin-down electrons in the $\mathrm{AF}$ ground state of $\mathrm{CrC}_{4} \mathrm{Cr}$, namely spin-valve is off. In its FM excited state while the transmission of spindown electrons is substantially suppressed, the conductance of spin-up electrons is enhanced by one order of magnitude. In the latter situation, the spin valve is on. This is a clear indication of spin-valve effect through the linear $\mathrm{CrC}_{4} \mathrm{Cr}$ molecule between two gold electrodes.

In Fig 13, a behavior similar to $\mathrm{CrC}_{n} \mathrm{Cr}$ molecules is found for $\mathrm{CoC}_{n} \mathrm{Co}$ molecules between two gold-electrodes. Here the spin valve effect occurs for $n=3$ and $n=5$. In the excited FM state of $\mathrm{CoC}_{3} \mathrm{Co}$ molecule the conductance of minority spin states is enhanced. We note that spin-valve effect in $\mathrm{CrC}_{4} \mathrm{Cr}$ and $\mathrm{CoC}_{3} \mathrm{Co}$ occurs effectively at $E<E_{F}$, and at $E=E_{F}$ for $\mathrm{CoC}_{5} \mathrm{Co}$. The shift of the maximum transmission from the Fermi level arises due to the shift of the energy levels of $\mathrm{TM}-\mathrm{C}_{n}-\mathrm{TM}$ molecule due to various reasons, such as number of $\mathrm{C}$ atoms and coupling to electrodes. We also note that the contribution of tunneling becomes significant for small $n$.

It should be noted that the most fundamental finding of the present work is the regular alternation of the ground state of $\mathrm{CoC}_{n} \mathrm{Co}$ atomic chains (and also other $\mathrm{TM}-\mathrm{C}_{n}$-TM atomic chains with $\mathrm{TM}=\mathrm{Ti}, \mathrm{V}, \mathrm{Mn}, \mathrm{Cr}, \mathrm{Fe}, \mathrm{Mo}$ ) between FM and $\mathrm{AF}$ states as $n$ is varied. Stated differently, for example, AF ground state of such a chain changes to FM ground state and vice versa when $n$ is increased or decreased by one. Calculations of similar chain structures have been recently reported by another group, ${ }^{62,63}$ but our results do not confirm some of their conclusions. They analyze spin-dependent energetics and conductance for 1D atomic carbon wires consisting of terminal magnetic (Co) and interior nonmagnetic 
(C) atoms sandwiched between gold electrodes, obtained by employing first-principles gradient-corrected density functional theory and Landauer's formalism for conductance. They report that the antiparallel spin configuration of the two terminal Co atoms corresponds to the ground state irrespective of the number of $\mathrm{C}$ atoms in the wire for $n$ up to 13 .

In systems with open-shell configurations it is difficult to converge to the ground state. ${ }^{64}$ This is why in well developed commercial codes (similar to GAUSSIAN03), imposing wave function stability is highly recommended to achieve the ground state for an open-shell configuration. Unfortunately, this subtle point is sometimes overlooked. However, we knew that our investigated systems would have several local minima and would be difficult to converge to the ground state. Hence each system (presented in the paper) has undergone stringent criteria. They have been simulated starting from different initial conditions. We have also tested our conclusions by employing three independent codes, VASP, SIESTA, and GAUSSIAN03. In the latter code we have also imposed wave function stability on our systems. All these extensive numerical tests led to consistent results which disagree with the conclusions of Refs. 62 and 63 . We also note a striking example which clearly shows that the calculations of Ref. 62 did not converge. The conductance results presented in Fig. 5 of the paper by Pati et al. ${ }^{62}$ for the AF state of a symmetric molecule are unrealistic. Due to the system symmetry spin-up conductance should match exactly to that of spin-down; however, their results do not reflect this simple criterion. Based on these arguments we believe that the results of Ref. 62 did not converge to the ground states of the structures. Hence they miss the odd- and even- $n$ disparity observed in our work.

\section{CONCLUSIONS}

We have studied the spin-dependent electronic, magnetic, and transport properties of atomic chain structures composed of carbon and transition metals, $\mathrm{TM}^{-\mathrm{C}_{n}}$ - $\mathrm{TM}$ molecules, using first-principles methods. Synthesis of the linear structures of these finite atomic chains is energetically feasible, and they are stable even at elevated temperatures. The indirect exchange interaction of the TM atoms mediated by the carbon atomic chain determines whether the FM or AF alignment of atomic moments leads to a lower energy. The ground state configuration and the total magnetic moment of the structures are determined by the number of carbon atoms in the spacer, and the type of the TM atom. The size-dependent variations of the physical properties of such systems exhibit several distinct forms, including regular alternations for $\mathrm{Ti}$, $\mathrm{V}, \mathrm{Mn}, \mathrm{Cr}, \mathrm{Fe}$, and $\mathrm{Co}$, and some irregular forms for $\mathrm{Sc}$ and $\mathrm{Ni}$ cases. In order to understand the underlying mechanism of such diverse variations we presented a simple tightbinding model. We also investigated the transport properties of these structures. The conductance of $\mathrm{TM}^{-} \mathrm{C}_{n}-\mathrm{TM}$ molecules when connected to metallic electrodes shows a strong spin-valve effect.

\section{ACKNOWLEDGMENTS}

S.C. and R.T.S. acknowledge partial financial support from TÜBA and TÜBA/GEBİ, respectively.
*Electronic address: ciraci@fen.bilkent.edu.tr

${ }^{1}$ G. A. Prinz, Science 282, 1660 (1998).

${ }^{2}$ S. A. Wolf, D. D. Awschalom, R. A. Buhrman, J. M. Daughton, S. von Molnar, M. L. Roukes, A. Y. Chtchelkanova, and D. M. Treger, Science 294, 1488 (2001).

${ }^{3}$ I. Zutic, J. Fabian, and S. Das Sarma, Rev. Mod. Phys. 76, 323 (2004).

${ }^{4}$ Magnetic Multilayers and Giant Magnetoresistance, edited by U. Hartman (Springer, Berlin, 2000).

${ }^{5}$ E. Hirota, H. Sakakima, and K. Inomata, Giant MagnetoResistance Devices (Springer, Berlin, 2002).

${ }^{6}$ P. M. Levy and I. Mertig, in Spin Dependent Transport in Magnetic Nanostructures, edited by S. Maekawa and T. Shinjo (Taylor and Francis, New York, 2002), pp. 47-111.

${ }^{7}$ M. N. Baibich, J. M. Broto, A. Fert, F. Nguyen Van Dau, F. Petroff, P. Etienne, G. Creuzet, A. Friederich, and J. Chazelas, Phys. Rev. Lett. 61, 2472 (1988); G. Binasch, P. Grünberg, F. Saurenbach, and W. Zinn, Phys. Rev. B 39, 4828 (1989).

${ }^{8}$ P. Bruno, Phys. Rev. B 52, 411 (1995).

${ }^{9}$ M. D. Stiles, J. Magn. Magn. Mater. 200, 322 (1999).

${ }^{10}$ S. S. P. Parkin, N. More, and K. P. Roche, Phys. Rev. Lett. 64, 2304 (1990).

${ }^{11}$ D. M. Edwards, J. Mathon, R. B. Muniz, and M. S. Phan, Phys. Rev. Lett. 67, 493 (1991).

${ }^{12}$ J. d'Albuquerque e Castro, M. S. Ferreira, and R. B. Muniz, Phys.
Rev. B 49, 16062 (1994).

${ }^{13}$ A. T. Costa, D. F. Kirwan, and M. S. Ferreira, Phys. Rev. B 72, 085402 (2005).

${ }^{14}$ V. B. Shenoy, Phys. Rev. B 71, 125431 (2005).

${ }^{15}$ E. G. Emberly and G. Kirczenow, Chem. Phys. 281, 311 (2002).

${ }^{16}$ Y. Wei, Y. Xu, J. Wang, and H. Guo, Phys. Rev. B 70, 193406 (2004).

${ }^{17}$ A. R. Rocha, V. M. García-Suárez, S. W. Bailey, C. J. Lambert, J. Ferrer, and S. Sanvito, Nat. Mater. 4, 335 (2005).

${ }^{18}$ H. Dalgleish and G. Kirczenow, Phys. Rev. B 72, 184407 (2005).

${ }^{19}$ D. Waldron, P. Haney, B. Larade, A. MacDonald, and H. Guo, Phys. Rev. Lett. 96, 166804 (2006).

${ }^{20}$ A. R. Rocha, V. M. García-Suárez, S. Bailey, C. Lambert, J. Ferrer, and S. Sanvito, Phys. Rev. B 73, 085414 (2006).

${ }^{21}$ J.-L. Mozos, C. C. Wan, G. Taraschi, J. Wang, and H. Guo, Phys. Rev. B 56, R4351 (1997).

${ }^{22}$ P. Sen, S. Ciraci, A. Buldum, and I. P. Batra, Phys. Rev. B 64, 195420 (2001).

${ }^{23}$ B. Larade, J. Taylor, H. Mehrez, and H. Guo, Phys. Rev. B 64, 075420 (2001).

${ }^{24}$ N. Agrait, C. Untiedt, G. Rubio-Bollinger, and S. Vieira, Phys. Rev. Lett. 88, 216803 (2002).

${ }^{25}$ F. J. Ribeiro and M. L. Cohen, Phys. Rev. B 68, 035423 (2004).

${ }^{26}$ F. Picaud, V. Pouthier, C. Girardet, and E. Tosatti, Surf. Sci. 547, 249 (2003). 
${ }^{27}$ S. Okano, K. Shiraishi, and A. Oshiyama, Phys. Rev. B 69, 045401 (2004)

${ }^{28}$ E. Z. da Silva, F. D. Novaes, A. J. R. da Silva, and A. Fazzio, Phys. Rev. B 69, 115411 (2004).

${ }^{29}$ S. Tongay, S. Dag, E. Durgun, R. T. Senger, and S. Ciraci, J. Phys.: Condens. Matter 17, 3823 (2005).

${ }^{30}$ R. T. Senger, S. Tongay, S. Dag, E. Durgun, and S. Ciraci, Phys. Rev. B 71, 235406 (2005).

${ }^{31}$ R. T. Senger, S. Tongay, E. Durgun, and S. Ciraci, Phys. Rev. B 72, 075419 (2005).

${ }^{32}$ S. R. Bahn and K. W. Jacobsen, Phys. Rev. Lett. 87, 266101 (2001).

${ }^{33}$ A. Smogunov, A. Dal Corso, and E. Tosatti, Phys. Rev. B 70, 045417 (2004).

${ }^{34}$ M. Wierzbowska, A. Delin, and E. Tosatti, Phys. Rev. B 72, 035439 (2005)

${ }^{35}$ A. Smogunov, A. Dal Corso, and E. Tosatti, Phys. Rev. B 73, 075418 (2006).

${ }^{36}$ R. A. de Groot, F. M. Mueller, P. G. van Engen, and K. H. J. Buschow, Phys. Rev. Lett. 50, 2024 (1983).

${ }^{37}$ W. E. Pickett and J. S. Moodera, Phys. Today 54, 39 (2001).

${ }^{38}$ H. Akinaga, T. Manago, and M. Shirai, Jpn. J. Appl. Phys., Part 2 39, L1118 (2000).

${ }^{39}$ C. Y. Fong, M. C. Qian, J. E. Pask, L. H. Yang, and S. Dag, Appl. Phys. Lett. 84, 239 (2004).

${ }^{40}$ S. Dag, S. Tongay, T. Yildirim, E. Durgun, R. T. Senger, C. Y. Fong, and S. Ciraci, Phys. Rev. B 72, 155444 (2005).

${ }^{41}$ M. C. Payne, M. P. Teter, D. C. Allen, T. A. Arias, and J. D. Joannopoulos, Rev. Mod. Phys. 64, 1045 (1992).

${ }^{42}$ Numerical computations have been carried out by using VASP software: G. Kresse and J. Hafner, Phys. Rev. B 47, 558 (1993); G. Kresse and J. Furthmuller, ibid. 54, 11169 (1996).

${ }^{43}$ W. Kohn and L. J. Sham, Phys. Rev. 140, A1133 (1965); P. Hohenberg and W. Kohn, Phys. Rev. 136, B864 (1964).

${ }^{44}$ D. Vanderbilt, Phys. Rev. B 41, R7892 (1990).
${ }^{45}$ J. P. Perdew, K. Burke, and M. Ernzerhof, Phys. Rev. Lett. 77, 3865 (1996).

${ }^{46}$ S. A. Adelman and J. D. Doll, J. Chem. Phys. 64, 2375 (1976).

${ }^{47}$ P. Ordejón, E. Artacho, and J. M. Soler, Phys. Rev. B 53, R10441 (1996); J. M. Soler, E. Artacho, J. D. Gale, A. Garcia, J. Junquera, P. Ordejon, and D. Sanchez-Portal, J. Phys.: Condens. Matter 14, 2745 (2002).

${ }^{48}$ Gaussian03, Revision C. 02, M. J. Frisch et al., Gaussian Inc., Wollingford CT, 2004.

${ }^{49}$ S. Datta, Electron Transport in Mesoscopic Systems (Cambridge University Press, Cambridge, 1997).

${ }^{50}$ M. B. Nardelli, Phys. Rev. B 60, 7828 (1999).

${ }^{51}$ S. Tongay, R. T. Senger, S. Dag, and S. Ciraci, Phys. Rev. Lett. 93, 136404 (2004).

${ }^{52} \mathrm{G}$. Roth and H. Fischer, Organometallics 15, 5766 (1996).

${ }^{53}$ S. Eisler et al., J. Am. Chem. Soc. 127, 2666 (2005).

${ }^{54}$ X. Zhao, Y. Ando, Y. Liu, M. Jinno, and T. Suzuki, Phys. Rev. Lett. 90, 187401 (2003).

${ }^{55}$ D. M. Ceperley and B. J. Alder, Phys. Rev. Lett. 45, 566 (1980).

${ }^{56}$ N. D. Lang and Ph. Avouris, Phys. Rev. Lett. 81, 3515 (1998); 84, 358 (2000).

${ }^{57}$ N. D. Lang, Phys. Rev. B 52, 5335 (1995); Phys. Rev. Lett. 79, 1357 (1997).

${ }^{58}$ M. A. Ruderman and C. Kittel, Phys. Rev. 96, 99 (1954); T. Kasuya, Prog. Theor. Phys. 16, 45 (1956); T. Kasuya, ibid. 16, 58 (1956); K. Yosida, Phys. Rev. 106, 893 (1957).

${ }^{59}$ P. Bruno, J. Magn. Magn. Mater. 121, 238 (1993).

${ }^{60}$ P. Bruno, Phys. Rev. B 52, 411 (1995).

${ }^{61}$ M. D. Stiles, Phys. Rev. B 48, 7238 (1993).

${ }^{62}$ R. Pati, M. Mailman, L. Senapati, P. M. Ajayan, S. D. Mahanti, and S. K. Nayak, Phys. Rev. B 68, 014412 (2003).

${ }^{63}$ L. Senapati, R. Pati, M. Mailman, and S. K. Nayak, Phys. Rev. B 72, 064416 (2005).

${ }^{64}$ R. Seeger and J. A. Pople, J. Chem. Phys. 66, 3045 (1977). 